10.1515/astro-2017-0317

Baltic Astronomy, vol.20, 448-452, 2011

\title{
THE COMPLEX BROAD ABSORPTION LINE PROFILES IN A SAMPLE OF QSO SPECTRA
}

\author{
E. Lyratzi ${ }^{1,2}$, E. Danezis ${ }^{1}$, L. Č. Popović ${ }^{3}$, A. Antoniou ${ }^{1,4}$, M. S. Dimitrijevićc ${ }^{3,5}$ \\ and D. Stathopoulos ${ }^{1,2}$ \\ 1 University of Athens, Faculty of Physics Department of Astrophysics, Astron- \\ omy and Mechanics, Panepistimioupoli, Zographou 157 84, Athens, Greece \\ 2 Eugenides Foundation, 387 Sygrou Av., 17564, Athens, Greece \\ 3 Astronomical Observatory of Belgrade, Volgina 7, 11160 Belgrade, Serbia \\ 4 University of Peloponnese, Faculty of Science and Technology, Department of \\ Telecommunications Science and Technology, Karaiskakis Str. 22100 Tripolis, \\ Greece \\ 5 Laboratoire d'Étude du Rayonnement et de la Matiére en Astrophysique, Ob- \\ servatoire de Paris-Meudon, UMR CNRS 8112, Bâtiment 18, 5 Place Jules \\ Janssen, F-92195 Meudon Cedex, France
}

Received: 2011 August 8; accepted: 2011 August 15

\begin{abstract}
Most of the Broad Absorption Lines (BALs) in quasars show very complex profiles. An idea to explain these profiles is that the dynamical systems of broad line regions are not homogeneous but consist of a number of dense regions or ion populations with different physical parameters. This approach is used to study the ultraviolet C IV resonance lines in the spectra of a group of high ionization BAL quasars, using the Gauss-Rotation model.
\end{abstract}

Key words: quasars: absorption lines

\section{INTRODUCTION}

Approximately $10 \%$ of all quasars exhibit broad, blue-shifted absorption lines. The outflow velocity of the regions producing these lines can reach $(0.1-0.2) c$. Usually, in their spectra we observe the lines of atoms with high ionization, such as C IV at $\lambda=1549 \AA$, Si IV at $\lambda=1397 \AA$, N V at $\lambda=1240 \AA$ and Ly $\alpha$. In some cases, low ionization lines, such as Mg II at $\lambda=2798 \AA$ and AlIII at $\lambda=1857 \AA$, also exhibit broad absorption lines (see, e.g., Hamann et al. 1993; Crenshaw et al. 2003). These Broad Absorption Lines (BALs) have different shapes; some types of these objects may also have differences in their continua (Reichard et al. 2003).

The spectra of the BAL quasars usually are interpreted as a combination of (1) a broad-band continuum arising from the central engine, (2) broad emission lines coming from the broad emission line regions (usually designated as BLRs or BELRs), emerging near the quasar center, and (3) superposed broad absorption lines, originating in a separate outlying region, so called Broad Absorption Line Region (BALR) (see also Lyratzi et al. 2009). However, it is also possible, that 
both the emission and absorption lines originate in the same line-forming region (Branch et al. 2002).

An important question is: what is the physical connection between the standard BLRs and BALRs? It is known that at least a part of BLRs originate in the wind outflowing from the accretion disks (see Murray \& Chiang 1998; Popović et al. 2004).

Another question is: where are BALRs placed with respect to the quasar centers and BLRs? To answer this question, one should investigate kinematical properties of the emission and absorption lines.

Disk wind models (Murray \& Chiang 1998; Proga et al. 2000; Williams et al. 1999) explain many properties of BAL quasars, but it is unclear if they can explain the full range of BAL profiles and column densities. BALs are caused by outflowing gas intrinsic to the quasar, and are not produced by galaxies along the line of sight (as is the case for most narrow-absorption systems).

Determining whether a quasar contains a BALR is a complicated task. The standard method is to calculate the 'balnicity' index (BI), defined by Weymann et al. (1991).

Here we present some ideas directed to explain the complex structure of BALs in quasars and investigate the ultraviolet C IV resonance lines in the spectra of a group of high ionization BAL (Hi-BAL) quasars using the Gauss-Rotation model (GR model).

\section{THE MULTI-STRUCTURE OF BALs}

Most of BALs in quasars exhibit very complex profiles. This means that we cannot fit them with a known physical distribution. An idea is that the dynamical systems of BLRs are not homogeneous but consist of a number of dense regions or ion populations with different physical parameters.

Each of these dense regions gives an independent classical absorption line. If the source regions rotate with a large velocity and move radially with lower velocities, the produced lines have large widths and small shifts. As a result, they are blended among themselves as well as with the main spectral lines forming a complicated profiles. A similar model is used to explain the complex profiles of broad lines in the spectra of hot emission-line stars (Danezis et al. 2006, 2007; Lyratzi et al. 2009). Based on this idea, we study BALs by the ultraviolet CIV resonance lines in the spectra of $30 \mathrm{Hi}$-BAL QSOs using the GR model (Danezis et al. 2006, 2007; Lyratzi et al. 2009).

We assume that broad emission and absorption line regions are composed of a number of successive independent absorbing/emitting density layers of matter originating in the disk wind. The absorbing regions have three apparent velocities (projected on the line-of-sight of an observer): radial velocity $\left(\mathrm{V}_{\text {rad }}\right)$ of the BALR, random velocity of the ions $\left(\mathrm{V}_{\text {rand }}\right)$ in the BALR and the rotational velocity $\left(\mathrm{V}_{\text {rot }}\right)$ of the BALR.

\section{DATA AND SPECTRAL ANALYSIS}

In order to study the C IV resonance lines (1548.187 and $1550.772 \AA$ ) we apply the GR model to the spectra of 30 Broad Absorption Line Quasars (BAL QSOs) taken from the Sloan Digital Sky Survey (SDSS) Data Release 7. The SDSS imaging survey uses a wide-field multi-CCD camera (Gunn et al. 1998). The 

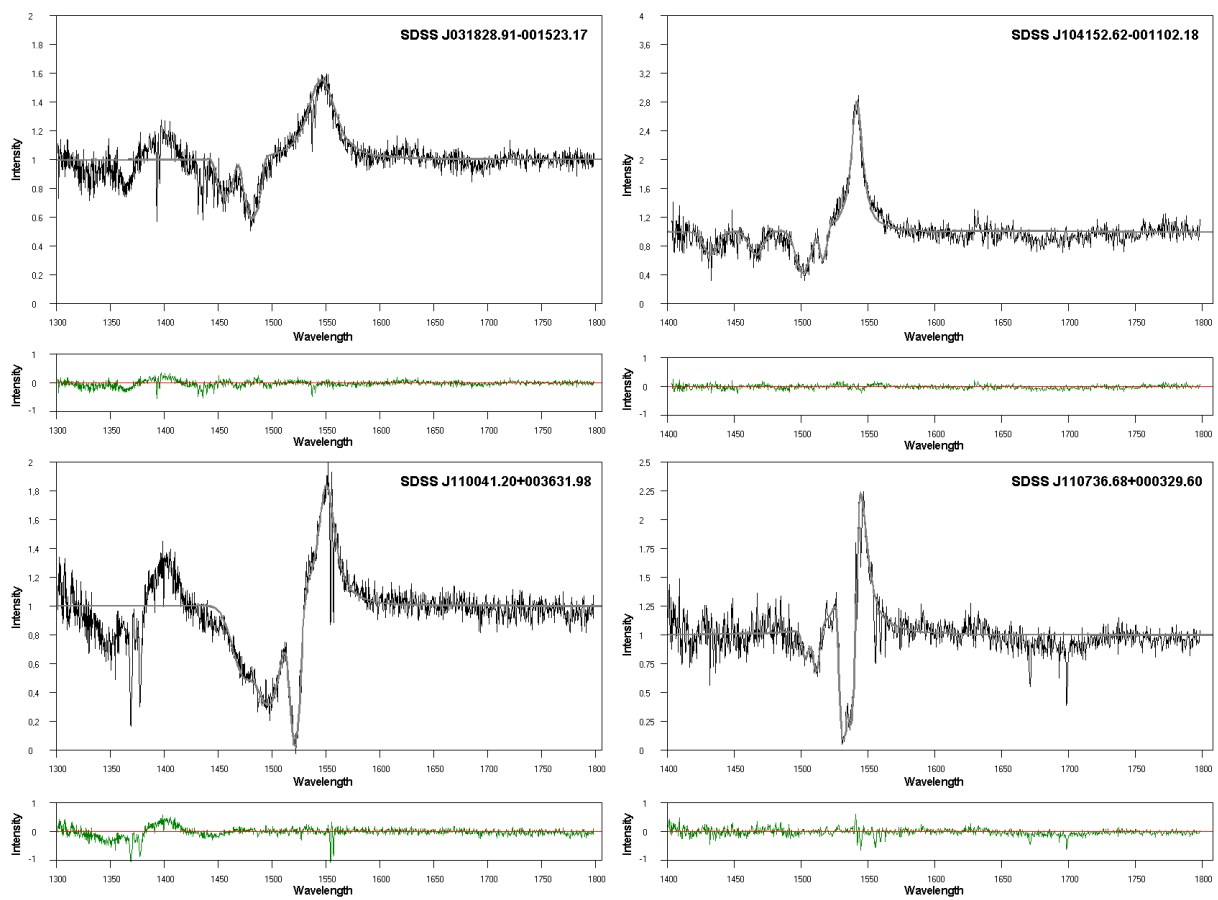

Fig. 1. Four examples of the fitted spectra. Black lines show the observed spectra and grey lines - theoretical line profiles given by the GR model. The lower panels show differences between the observed spectra and the corresponding theoretical profiles.

spectra cover the optical range 3800-9200 $\AA$ at a resolution of $1800-2100$.

In Figure 1 we give some examples of the fitted spectra. The black line corresponds to the observed spectra and the grey line corresponds to the theoretical line profiles given by the GR model. The lower lines present the residuals, i.e., the differences between the observed spectrum and the theoretical profile.

We studied all the absorption lines that follow the first Balnicity Index criterion, i.e. the lines that are located between 3000 and $25000 \mathrm{~km} / \mathrm{s}$ blueward of the CIV emission, what means that these lines come from the studied BAL quasars. Then, from all these lines we accepted as BALs only those which follow the rest Balnicity Index criteria, i.e., the absorption lines are at least $2000 \mathrm{~km} / \mathrm{s}$ broad and for them the absorption falls at least $10 \%$ below the continuum. We found that 25 of the 30 quasars of our sample have broad absorption lines.

\section{RESULTS AND CONCLUSIONS}

Using the GR model, we were able to fit the complex profiles of the studied C IV lines with 1 to 5 absorption components. For them we calculated the values of the kinematical parameters (rotational, radial and random velocities), as well as the column density and the absorbed energy. The calculated values of kinematical parameters only, are given in Tables 1 and 2.

It seems that the dynamical systems of BLRs are not homogeneous but consist of a number of density regions or ion populations with different physical parameters. Each one of these density regions gives us an independent classical absorption 
Table 1. Radial velocities $(\mathrm{km} / \mathrm{s})$.

\begin{tabular}{l|rrrrr}
\hline Object name (SDSS) & $V_{\text {rad1 }}$ & $V_{\text {rad2 }}$ & $V_{\text {rad3 }}$ & $V_{\text {rad4 }}$ & $V_{\text {rad5 }}$ \\
\hline J001025.90+005447.6 & -15092 & -10642 & & & \\
J001438.28-010750.1 & -17026 & & & & \\
J001502.26+001212.4 & -19348 & & & & \\
J002127.88+010420.1 & -14624 & & & -6192 & \\
J003551.98+005726.4 & & & & & \\
J004041.39-005537.3 & -17220 & & -7740 & & \\
J004118.59+001742.4 & -13931 & & & & \\
J004323.43-001552.4 & -20896 & -12770 & & & \\
J004732.73+002111.3 & -17414 & & & & \\
J005355.15-000309.3 & -17026 & -11609 & & & -4063 \\
J005419.99+002727.9 & -17607 & & -8513 & & \\
J010241.04-004208.9 & -17414 & -11802 & & & -4063 \\
J010336.40-005508.7 & -17800 & & & -6385 \\
J011227.60-011221.7 & -21283 & -16446 & -8513 & -6385 & \\
J015024.44+004432.99 & & & -7546 & & -3483 \\
J015048.83+004126.29 & -18381 & & & -5321 & -3096 \\
J021327.25-001446.92 & & & & \\
J023908.99-002121.42 & -17414 & & -8513 & & \\
J025747.75-000502.91 & & & -7352 & & \\
J031828.91-001523.17 & -17994 & -13157 & & & \\
J102517.58+003422.17 & -17994 & & & & \\
J104109.86+001051.76 & & & -9867 & & \\
J104152.62-001102.18 & -22637 & -16059 & -9287 & & \\
J104841.03+000042.81 & -19348 & & & & \\
J110041.20+003631.98 & -14704 & -10061 & & -5417 & \\
\hline Mean & -17757 & -12818 & -8416 & -5829 & -3676 \\
\hline
\end{tabular}

line.

As we can see in Tables 1 and 2, the GR model is able to reproduce accurately the complex profiles of Hi-BAL QSOs and to calculate a group of physical parameters of the corresponding BALRs.

The absorption lines which follow the BI criteria satisfy the following conclusions: (1) their radial velocities are between $-3676 \pm 410 \mathrm{~km} / \mathrm{s}$ and $-17757 \pm 2171$ $\mathrm{km} / \mathrm{s}$; (2) their random velocities are between $1117 \pm 302 \mathrm{~km} / \mathrm{s}$ and $2952 \pm 1704$ $\mathrm{km} / \mathrm{s}$; (3) the column density of all the absorption components of both doublet lines is almost the same, with the mean value $-3.35 \times 10^{-10} \pm 0.24 \times 10^{-10} \mathrm{~cm}^{-2}$; (4) the absorbed energy is $-5.52 \pm 1.32 \mathrm{eV}$ for $\lambda 1548.187 \AA$ and $-4.79 \pm 1.26 \mathrm{eV}$ for $\lambda 1550.772 \AA$.

ACKNOWLEDGMENTS. This research project is in progress at the University of Athens, Department of Astrophysics, Astronomy and Mechanics; we are thankful to the Special Account for Research Grants for the financial support.

This work was also supported by the Ministry of Science and Technological Development of Serbia through the projects "Influence of coalitional processes on astrophysical plasma line shapes" and "Astrophysical spectroscopy of extragalactic objects". We also acknowledge the use of the data from the SDSS and SDSS-II surveys. 
Table 2. Rotational and random velocities $(\mathrm{km} / \mathrm{s})$.

\begin{tabular}{|c|c|c|c|c|c|c|c|c|c|c|}
\hline Object name (SDSS) & $V_{\text {rot1 }}$ & $V_{\text {rot2 }}$ & $V_{\text {rot3 }}$ & $V_{\text {rot4 }}$ & $V_{\text {rot5 }}$ & $V_{\text {rand1 }}$ & $V_{\text {rand2 }}$ & $V_{\text {rand3 }}$ & $V_{\text {rand4 }} \mathrm{I}$ & $V_{\text {rand5 }}$ \\
\hline J001025.90+005447.6 & 600 & 600 & & & & 1596 & 1368 & & & \\
\hline J001438.28-010750.1 & 100 & & & & & 6155 & & & & \\
\hline J001502.26+001212.4 & 100 & & & & & 6155 & & & & \\
\hline J002127.88+010420.1 & 100 & & & & & 6155 & & & & \\
\hline J003551.98+005726.4 & & & & 100 & & & & & 787 & \\
\hline J004041.39-005537.3 & 3000 & & & & & 4559 & & & & \\
\hline J004118.59+001742.4 & 100 & & 100 & & & 2508 & & 1824 & & \\
\hline J004323.43-001552.4 & 3000 & 3000 & & & & 2736 & 2736 & & & \\
\hline J004732.73+002111.3 & 100 & & & & & 4332 & & & & \\
\hline J005355.15-000309.3 & 100 & 100 & & & & 912 & 912 & & & \\
\hline J005419.99+002727.9 & 3000 & & 550 & & & 2280 & & 2052 & & \\
\hline J010241.04-004208.9 & 50 & 50 & & & 50 & 1824 & 1824 & & & 798 \\
\hline J010336.40-005508.7 & 100 & & & & 100 & 3420 & & & & 1596 \\
\hline J011227.60-011221.7 & 550 & 550 & 550 & 550 & & 1596 & 1254 & 912 & 798 & \\
\hline J015024.44+004432.99 & & & 1500 & & 2200 & & & 1368 & & 1140 \\
\hline $\mathrm{J} 015048.83+004126.29$ & 50 & & & & & 4104 & & & & \\
\hline J021327.25-001446.92 & & & & 100 & 100 & & & & 912 & 935 \\
\hline J023908.99-002121.42 & 30 & & 30 & & & 2280 & & 2280 & & \\
\hline J025747.75-000502.91 & & & 100 & & & & & 2280 & & \\
\hline J031828.91-001523.17 & 2000 & 2000 & & & & 456 & 570 & & & \\
\hline $\mathrm{J} 102517.58+003422.17$ & 50 & & & & & 2508 & & & & \\
\hline $\mathrm{J} 104109.86+001051.76$ & & & 100 & & & & & 798 & & \\
\hline $\mathrm{J} 104152.62-001102.18$ & 50 & 50 & 50 & & & 1140 & 1140 & 1140 & & \\
\hline $\mathrm{J} 104841.03+000042.81$ & 50 & & & & & 2052 & & & & \\
\hline $\mathrm{J} 110041.20+003631.98$ & 50 & 70 & & 50 & & 2280 & 2280 & & 912 & \\
\hline Mean & | 1830 & 1538 & 550 & 550 & & 2952 & 1510 & 1582 & 852 & 1117 \\
\hline
\end{tabular}

\section{REFERENCES}

Branch D., Leighly K. M., Thomas R. C., Baron E. 2002, ApJ, 578, L37

Crenshaw M. D., Kraemer S. B., George I. M. 2003, ARA\&A, 41, 117

Danezis E., Popović L. Č., Lyratzi E., Dimitrijević M. S. 2006, AIPC, 876, 373

Danezis. E, Nikolaidis D., Lyratzi E. et al. 2007, PASJ, 59, 827

Gunn J. E., Carr M., Rockosi C. et al. 1998, AJ, 116, 3040

Hamann F., Korista K. T., Morris S. L. 1993, ApJ, 415, 541

Lyratzi E., Popović L. Č., Danezis E. et al. 2009, NewAR, 53, 179

Murray N., Chiang J. 1998, ApJ, 494, 125

Popović L. Č., Mediavilla E., Bon E., Ilić D. 2004, A\&A, 423, 909

Proga D., Stone J. M., Kallman T. R. 2000, ApJ, 543, 686

Reichard T. A., Richards G. T., Hall P. B. et al. 2003, AJ, 126, 2594

Weymann R. J., Morris S. L., Foltz C. B., Hewett P. C. 1991, ApJ, 373, 23

Williams R. J. R., Baker A. C., Perry J. J. 1999, MNRAS, 310, 913 